\title{
Reguladores en Conflicto: Cuando las urgencias ocultan las eficiencias
}

Eduardo Saavedra*

\section{Los mandatos confusos}

o múltiples en políticas

económicas pueden

generar serios conflictos de

interés en los reguladores

y llevar al abandono

de tareas primordiales

para el bienestar de la

sociedad, no por descuido

malintencionado, sino por

excesivo celo en unos pocos

deberes. Esto ocurre,

por ejemplo, cuando las

tareas políticas y las tareas

técnicas son ejecutadas

por un mismo ministerio

y se usa la regulación

de precios como medio

para cumplir las tareas

políticas.

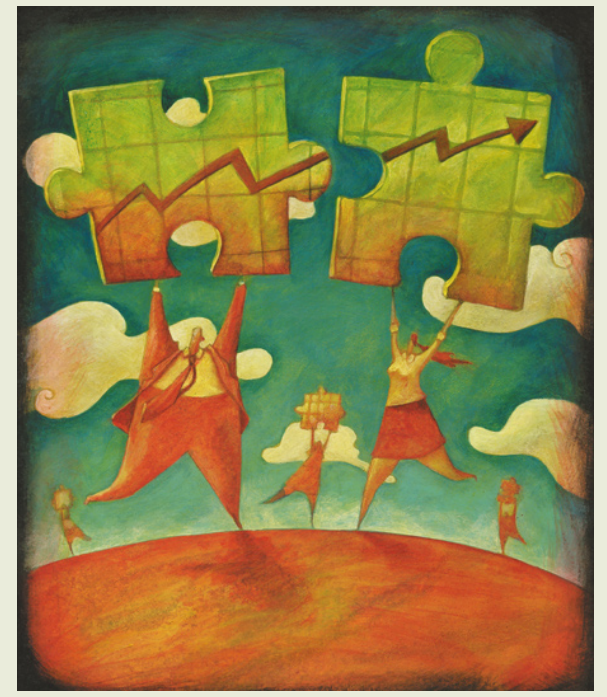

\section{Introducción}

Cuando se modificó el contrato laboral de los choferes de micros (uno que pagaba gran parte del sueldo como un porcentaje de los boletos cortados se cambió por uno de salario fijo), se pensaba que así se resolverían las locas carreras entre las micros, pero no se tomó en cuenta que un chofer con sueldo fijo perdería los incentivos para exigir el pago del servicio. La moraleja es que si como sociedad queremos que los usuarios paguen sus pasajes y además que el servicio no los exponga a riesgos innecesarios, no podemos esperar que el contrato laboral resuelva ambas tareas en conflicto. Si se opta por sueldos fijos, se requiere de otro instrumento para que conseguir que los pasajeros paguen el servicio, por ejemplo cobros automáticos o cámaras que vigilen que el chofer exija el pago.

El ejemplo nos muestra que es difícil pedirle a una persona que haga dos tareas a la vez, especialmente si quien solicita dichas misiones sólo se preocupa de verificar una de ellas. Este problemade múltiples tareas se resuelve muchas veces delegándolas en varias personas diferentes. Esto explica, por ejemplo, por qué un Presidente reparte entre varios ministros sus diversas políticas y separa a los ministerios gastadores (Salud, Educación, Obras Públicas, por ejemplo) del ministerio encargado de cuidar los recursos públicos (Hacienda).

Alinear los intereses de quienes desean hacer una tarea con los de quienes las ejecutan es complejo en la práctica, en especial para un gobierno que siempre tiene múltiples mandatos. 
De hecho, actualmente se observan serios conflictos de interés entre diversos objetivos que persiguen los reguladores en los sectores de energía, telecomunicaciones y concesiones de infraestructura.

\section{Política Energética}

Los temas energéticos de Chile, en particular las materias eléctricas, han estado en el tapete de la discusión pública desde hace una década. Primero fue la sequía de 1998 y 1999 , con cortes que afectaron a más del $90 \%$ de la población; luego dos cambios a la ley eléctrica y desde hace unos años el país sufre cortes de gas natural desde Argentina que han puesto en duda las ventajas de hacer negocios con nuestros vecinos. Y los temas seguirán, pues se habla de unas futuras plantas que regasificarán gas natural licuado y de nuevas políticas tendientes a favorecer el uso de energías renovables.

En este escenario, las tareas primordiales del gobierno en el ámbito energético son la eficiencia económica y la seguridad energética. La eficiencia económica busca que los precios de la energía entreguen señales correctas a los empresarios en materias de inversión (para que puedan invertir en plantas hidroeléctricas, térmicas, eólicas u otras), y que estos precios reflejen los costos de producción y no dejen excesivas rentas ni desfinancien a las empresas. La Comisión Nacional de Energía (CNE) es el brazo del gobierno encargado de velar porque las tarifas que paguen los usuarios sean económicamente correctas; la Superintendencia de Electricidad y Combustibles es el organismo encargado de velar por la calidad del servicio.

\section{Es difícil pedirle a}

una persona que haga

dos tareas a la vez, especialmente si quien solicita dichas misiones sólo se preocupa de verificar una de ellas

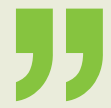

El objetivo de seguridad energética se refiere a la necesidad de que el país reduzca el riesgo de desabastecimiento de electricidad, combustibles líquidos y gas. Tal como en el ámbito personal todos estamos sujetos a riesgos como el de perder el empleo o enfermar, la sociedad está enfrentada a la incertidumbre de si habrá suficiente abastecimiento de energía. Los problemas de desabastecimiento energético en Chile provienen del hecho de que no tenemos reservas importantes de petróleo, carbón y gas natural, y que el recurso hídrico está sujeto a caprichos climáticos.

La gran dificultad que surge con la meta de la seguridad energética es que los gobernantes son (mucho) más enemigos del desabastecimiento que los propios ciudadanos: los cortes de luz ponen en serio peligro una reelección. El gobierno tiene un excesivo celo por reducir este riesgo, lo que eleva el precio que pagan los usuarios y, sobre todo, genera un conflicto entre los objetivos de seguridad energética y eficiencia económica: es más "barato" no subsidiar la mayor seguridad en forma directa sino que hacerlo por el camino oculto de distorsionar el sistema de precios, así nadie se entera.

En Chile ambos objetivos en conflicto están asignados a un mismo organismo, la CNE y en especial a su superior directo, el Ministro de Energía. La decisión de la Presidenta de la República de nombrar a un ministro con la tarea de hacer "todo lo posible para que la energía no falte y llegue en la cantidad necesaria para el desarrollo del país"1 oficializa una decisión política que se viene gestando desde hace un tiempo y que a pesar de ser un objetivo loable, abandona dos principios básicos sobre los cuales se ha sustentado la política energética de Chile en los últimos 25 años: i) es el sector privado quien decide sus tecnologías de inversión a partir de las 
señales que entregan directamente los precios y ii) los subsidios destinados a favorecer ciertos sectores o tecnologías son explícitos y directos. ${ }^{2}$

\section{La gran dificultad que}

surge con la meta de la

seguridad energética es

que los gobernantes son

(mucho) más enemigos

del desabastecimiento que

los propios ciudadanos:

\section{los cortes de luz ponen}

en serio peligro una

\section{reelección}

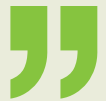

Como una mala idea nunca muere, hoy estamos frente a nuevas creaciones, el proyecto de ley de energías renovables y el de biocombustibles, que en nombre de la seguridad energética pretenden distorsionar el sistema de precios y esconder los subsidios que se requieren para financiar tecnologías alternativas por la vía de exigir un porcentaje mínimo de generación "verde". Si el objetivo de favorecer estas tecnologías fuera socialmente deseable, entonces la creación de un fondo específico que se licite termina por sincerar cuál es exactamente el objetivo de política perseguido, sea energético o ambiental, y además permite que la competencia por esos fondos gatille precios menores que los que resultan de imponer a la fuerza el uso de energías renovables.

Dado el mandato establecido al ministro al momento de su nombramiento, él parece no tener más opción que priorizar la seguridad energética. $\mathrm{Si}$ esto se hace además por la vía de sacar adelante proyectos como el de energías renovables o el de biocombustibles, esta priorización de tareas significaría un enorme retroceso para nuestra competitividad, el que no ha sido puesto de cara al país. El problema no es la falta de alternativas sino que al parecer la falta de credibilidad en el mercado estaría llevando a tomar decisiones al más alto nivel que en el nombre de la seguridad energética pueden terminar por destruir el otro objetivo fundamental: la búsqueda de la eficiencia económica.

Un adecuado diseño de la regulación en este sector pasa por reasignar tareas al interior del Ministerio de Energía. El ministro debe delegar las tareas tarifarias en la CNE, y guardar para sí las tareas relacionadas al objetivo de seguridad energética, que tiene muchos más elementos políticos que

\section{EXCELENTES PROFESIONALES, grandes personas

\section{Estudia Ingeniería Comercial e Ingeniería Comercial para Profesionales}

Almirante Barroso $\mathrm{N}^{\circ} 6$, Metro Los Héroes.

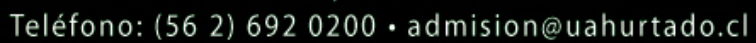

UNIVERSIDAD ALBERTO HURTADO LA UNIVERSIDAD JESUITA DE CHILE
FACULTAD DE ECONOMÍA Y NEGOCIOS 
técnicos. Las áreas dentro de la CNE que no sean regulatorias de precios deben depender directamente del ministro, así no se duplicarían nuevos organismos. De esta forma se exige al ministro que ambos objetivos sean prioridad ministerial, pero se le amarra de manos para no intervenir en las decisiones más técnicas en materia regulatoria al momento de querer resolver las tareas más políticas de su cartera.

\section{Regulación de las Telecomunicaciones}

Actualmente el regulador de este sector es la Subsecretaría de Telecomunicaciones, Subtel, organismo que tiene por misión: i) velar por el desarrollo del sector en Chile y ii) regular las tarifas de la empresa dominante en telefonía fija, los cargos de acceso de todas las empresas del sector (el precio que cobra una empresa por las llamadas a sus clientes desde clientes de otras empresas) y fiscalizar la entrega de los servicios de acuerdo a estándares de calidad definidos o aceptables.

Mientras el segundo objetivo es de regulación y fiscalización, el de velar por el desarrollo del sector de las telecomunicaciones es mucho más general, pues incluye el tutelar todas las políticas sectoriales. Es en esta segunda tarea en donde aparecen los conflictos, fundamentalmente porque la misión es poco clara: si se le exige a Subtel una meta específica en materia de penetración de las telecomunicaciones, el subsecretario utilizará todas las herramientas a su haber para lograr esa meta, aún a costa de afectar los objetivos de eficiencia económica que resultan de los roles de regulador y fiscalizador de esta subsecretaría.

En efecto: aun si las tareas de diseñar e implementar políticas y las de regular y fiscalizar son igualmente importantes para el Estado, ¿por qué el énfasis es desigual a la hora de tomar la mejor decisión? Si ambas grandes tareas están a cargo de una misma subsecretaría, el objetivo político opaca al de eficiencia económica, lo que no tiene nada de extraño pues, después de todo, al subsecretario lo evalúa el ministro o el Presidente de la República, quienes quieren llevar adelante la tarea de desarrollo del sector y están dispuestos a financiarlo aun a costa de distorsionar la eficiencia en las tarifas.

La telefonía móvil en Chile comenzó hace casi dos décadas, pero no es sino a fines de los noventa, cuando se introduce la tecnología PCS y la regla de "quien llama paga", que se hace evidente un sesgo desarrollista del sector. Es lamentable que el regulador no haya sido capaz de abstraerse de presiones políticas para sincerar precios con costos de proveer el servicio que difieren incluso entre comunas de una misma ciudad. El haber fijado tarifas similares dentro de una misma gran ciudad como Santiago, por ejemplo, lleva a la aparición de una amplia oferta de servicios de telecomunicaciones en Providencia,
Las Condes y Lo Barnechea y una oferta de servicios bastante menos prolífica en los otros barrios de la capital.

\section{Dado el mandato} establecido al ministro al momento de su nombramiento, él parece no tener más opción que priorizar la seguridad energética

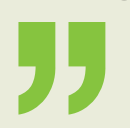

El problema de falta de decisiones políticas a la hora de modificar la ley de telecomunicaciones para adaptar la normativa de cargos de acceso a lo que la experiencia internacional y la literatura en el tema han clarificado, es otra muestra de que el status quo es contrario a la eficiencia económica. Nuevamente estamos frente a una situación en la que la indefinición legal permite fijar bajos cargos de acceso para la empresa dominante y altos cargos de acceso a pequeños competidores y a la telefonía móvil, lo que no solo permite la entrada ineficiente a esta industria y encarece los costos que finalmente pagan los usuarios, sino que además desfinancia a quien ya ha invertido en redes.

Este no es un tema de héroes y villanos. La actitud mostrada por Subtel en los 
últimos diez años es perfectamente consistente con el mandato que se le ha entregado y por el cual el subsecretario será evaluado. La tarea de aumentar la penetración de las telecomunicaciones se ha hecho bien si se observa que el porcentaje de personas con teléfonos móviles subió de $22 \%$ a $73 \%$, que se duplicaron los chilenos con acceso a
Internet y que la cobertura en telefonía fija estuvo estable entre 2000 y 2006.

Pero la consecuencia negativa de esta política también se puede palpar. Muchos de los usuarios que obtienen ahora una peor calidad de servicios, servicios con bajas inversiones en redes de telefonía fija y uso mayoritario

\section{Master of Arts in Economics}

\section{ILADES / GEORGETOWN UNIVERSITY}

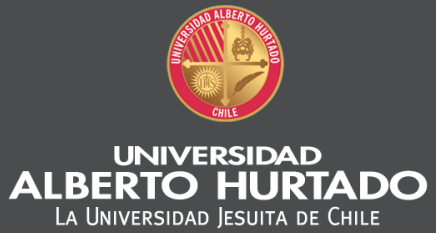

El Programa de Postgrado en Economía es conducente

al grado académico de "Master of Arts in Economics" (MAE),

que otorga Georgetown University y al grado de Magíster

en Economía que otorga la Universidad Alberto Hurtado.

El programa presenta tres variantes:

- Programa General

- Programa con Mención en Políticas Sociales (Public Economics)

- Programa con Mención

en Economía de Empresas (Business Economics). de teléfonos de prepago que los han vuelto a la época del "telégrafo", paradójicamente no tienen voz. Al final, estas personas son las más pobres y las menos escuchadas. Asimismo, esta política no ha aumentado el uso de la telefonía, solo ha sustituido el modo en que las personas se comunican, pues hoy se cursa la misma cantidad de minutos llamados que hace 15 años, pero se paga en promedio mucho más por ese servicio (aunque, ciertamente, se reciben otros servicios adicionales, como la movilidad).

\section{Es lamentable que el}

\section{regulador no haya sido}

capaz de abstraerse de

presiones políticas para

sincerar precios con costos

\section{de proveer el servicio}

\section{que difieren incluso entre}

\section{comunas de una misma}

\section{ciudad}

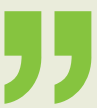

Estamos de nuevo frente a un tema de conflicto de interés en la autoridad política. La solución pasa por separar las tareas más técnicas de regulación y fiscalización de aquellas más políticas, lo que se resuelve 


\section{Observatorio Económico}

creando una Superintendencia de Telecomunicaciones. Al igual que en energía, no se requiere agrandar innecesariamente el tamaño del Estado sino que bastaría con mover las plantas dedicadas a regulación tarifaria y fiscalización desde Subtel hacia esta nueva superintendecia ${ }^{3}$.

\section{Concesiones de Infraestructura}

El fuerte desarrollo de las obras públicas en Chile en los últimos diez años está fuertemente relacionado con el programa de concesiones de carreteras, urbanas e interurbanas, que le han dado a nuestro país un aire de desarrollo impensado a principios de los noventa. Este fuerte desarrollo de las obras de infraestructura pública no es sólo de carreteras, sino también de puertos y aeropuertos, cárceles y empresas sanitarias, entre otras.

Los beneficios para el país son enormes, tanto en las comodidades para los usuarios que pagan por este servicio, las disminuciones en las pérdidas de vida por accidentes, el abaratamiento de los costos de transportes que hace más competitivas nuestras exportaciones y sobre todo los beneficios para los más desposeídos, al liberarse recursos fiscales para la política social. Parte importante de los éxitos de este programa de concesiones se debe a que el Ministerio de Obras Públicas creó la Unidad de Concesiones como un organismo especialmente dedicado a diseñar las obras a concesionar, con el objetivo muy claro de implementar un programa exitoso en atraer inversiones privadas.

\section{Un claro compromiso \\ de diseñar una \\ supertintendencia con}

poder para dialogar con

su contraparte y capaz

de hacer creíble que hará

cumplir los acuerdos

\section{comprometidos}

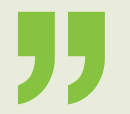

Dos problemas surgen del actual diseño de este programa: i) cómo manejar los incentivos que tienen los privados y el propio Estado para renegociar los contratos de concesión ya firmados, ii) cómo resolver los conflictos de interés que surgen de diseñar y adjudicar las concesiones exitosamente, con la misión de fiscalizar que las obras de inversión y la operación de estas concesiones se realicen de acuerdo a los criterios definidos. Ambos problemas pueden ser de gran impacto tanto para las concesiones ya adjudicadas, como para la credibilidad en los futuros proyectos de concesión.

El conflicto de interés en cómo manejar las renegociaciones con los inversionistas que ya se han adjudicado obras es mayúsculo, pues la renegociación de contratos pone un manto de dudas sobre el proceso de licitación y distorsiona las ofertas que los propios interesados proponen en ese momento, ya que finalmente no es la oferta económica la que cuenta sino la capacidad de lobby que tienen los proponentes para mover la balanza en su favor luego de que ellos y el gobierno quedan amarrados al firmar el contrato de concesión y realizar las inversiones pactadas.

Algunos ejemplos de este interés dañino por renegociar son las repetidas peticiones para revisar el contrato del túnel El Melón, las declaraciones del máximo ejecutivo de Tribasa a su llegada a Chile hace diez años (preguntó con quién y cómo se renegociaban los contratos de concesión justamente luego de haberse adjudicado tres tramos de la carretera Panamericana), o el deseo de algunos parlamentarios de abrir gratuitamente la Costanera Norte a las micros y a las motos para evitar una brecha aún mayor en la desigualdad de oportunidades entre ricos y pobres. ${ }^{4}$

\footnotetext{
La Subtel ha puesto a disposición del público un anteproyecto de ley en esta materia, el que puede consultarse en www.subtel.cl

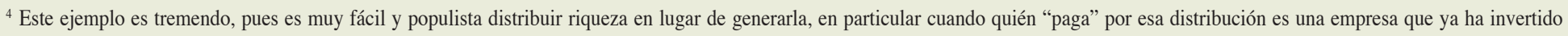
decenas de millones de dólares que sólo puede recuperar con los peajes.
} 
Si bien la Unidad de Concesiones ha creado los mecanismos de renegociación, algunas empresas concesionarias dicen que este mecanismo no ha funcionado adecuadamente pues no ha resuelto de manera expedita los problemas que se busca resolver y ha generado más espacios de conflicto que de encuentro. Lo mejor que puede hacer el MOP es poner un letrero en sus puertas diciendo "no se renegocia" y, por supuesto, cumplirlo. Las revisiones puntuales de contratos deben definitivamente sacarse de la Unidad de Concesiones.

\section{Se requiere dotar a los} reguladores técnicos de independencia política en sus acciones

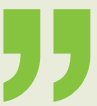

El segundo problema de conflicto que aparece con la institucionalidad de concesiones se refiere a la tarea de fiscalización de obras de inversión y de la operación de las concesiones ya adjudicadas. No podemos esperar que la tarea de concesionar -que pasa por atraer inversionistas, diseñar el proyecto de concesión en sus obras civiles, en las expropiaciones necesarias, en el diseño arquitectónico de acuerdo a requerimientos técnicos, económicos y hasta culturales- se vea opacada por las mismas personas ahora en un rol distinto: el de enfrentar a los mismos empresarios en la fiscalización de la calidad de las obras y del servicio entregado a los usuarios.

Estamos en este caso frente a un claro conflicto de interés. $\mathrm{Si}$ el policía tuviera por objetivo cuidar al automovilista, difícilmente podría multarlo por no cumplir las leyes del tránsito. Se requiere que el MOP traspase a una Superintendencia de Concesiones el rol de fiscalización de las concesiones ya otorgadas. Esto no solo pasa por un traspaso de personas desde una unidad a otra, sino también por un claro compromiso de diseñar una supertintendencia con poder para dialogar con su contraparte y capaz de hacer creíble que hará cumplir los acuerdos comprometidos. Se necesita, en consecuencia, modificar descripciones de cargos tan importantes como el de Inspector Fiscal, tarea que lleva a cabo una persona con un perfil de cargo excesivamente ingenieril, propio de las construcciones de obras, pero que no tiene relación con el cargo de un superintendente que vela por fiscalizar esos temas y además todos los otros relacionados con la calidad del servicio prestado.

\section{Conclusiones y Propuesta}

Los casos comentados en este informe se refieren a problemas de diseño y énfasis inconsistentes con la eficiencia económica en los sectores de energía, telecomunicaciones y concesiones de infraestructura. Estos son

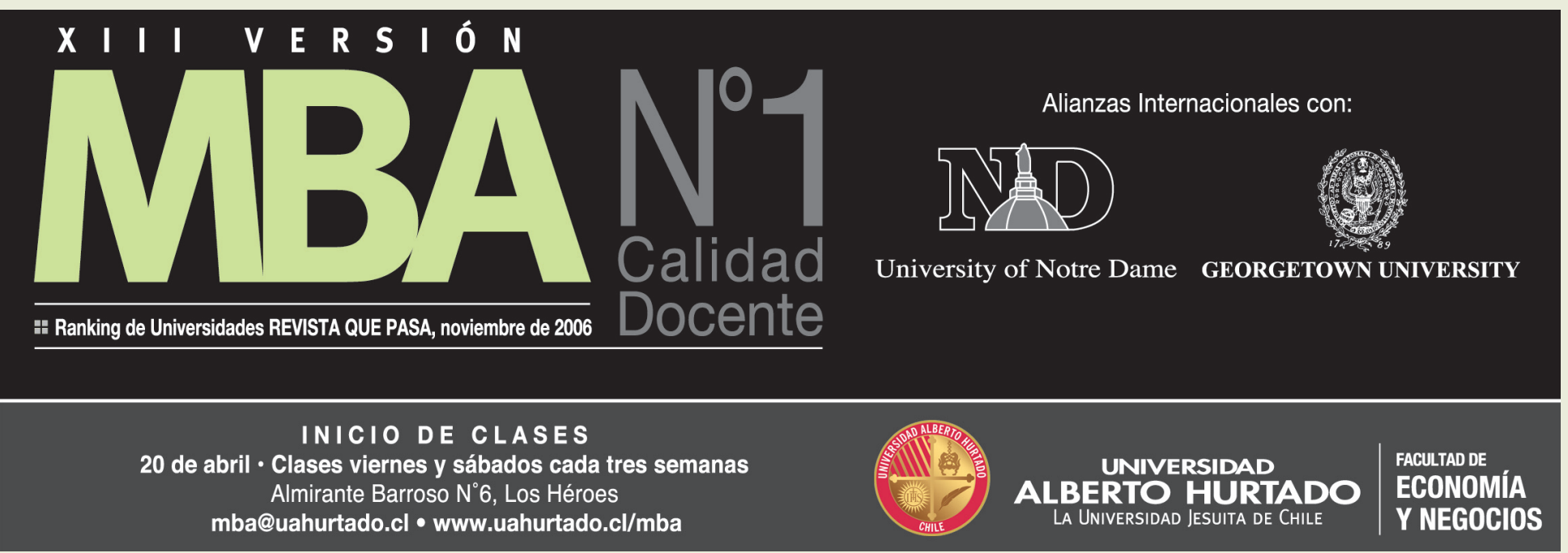




\section{Observatorio Económico}

sólo tres casos que pueden ampliarse, por ejemplo, a ciertas inconsistencias de objetivos que se observan en la regulación bancaria y de capitales, en educación, en salud, medioambiente y en la política social del gobierno.

La solución pasa por separar las tareas regulatorias de precios o de fiscalización de la calidad del servicio de las tareas más políticas de los respectivos ministerios. Obviamente, se requiere dotar a los reguladores técnicos de independencia política en sus acciones. La siguiente figura resume esta propuesta:
OBJETIVO POLÍTICO:

- Energía

- Telecomunicaciones

- Concesiones de Obras Públicas

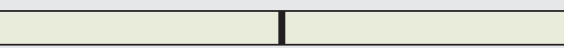

ORGANISMOS ENCARGADOS:

- Ministerio de Energía

- Subtel

- Unidad de Concesiones

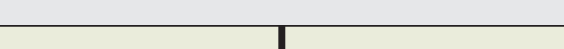

TAREAS ESPECÍFICAS:

- Seguridad Energética y velar por otros objetivos de la política

- Desarrollo Telecomunicaciones (acceso universal, país digital)

- Diseñar programas y licitar concesiones
OBJETIVO REGULATORIOTÉCNICO:

- Regulación de Precios

- Fiscalización

ORGANISMOS INDEPENDIENTES:

- Comisión Nacional de Energía, CNE

- Super. de Telecomunicaciones

- Superintendencia de Concesiones

TAREAS ESPECÍFICAS:

- Fijar tarifas (precio nudo referencial, transmisión y distribución)

- Fijar tarifas y cargos de acceso; fiscalizar calidad del servicio

- Fiscalizar cumplimiento de contratos (inversiones y calidad servicio)

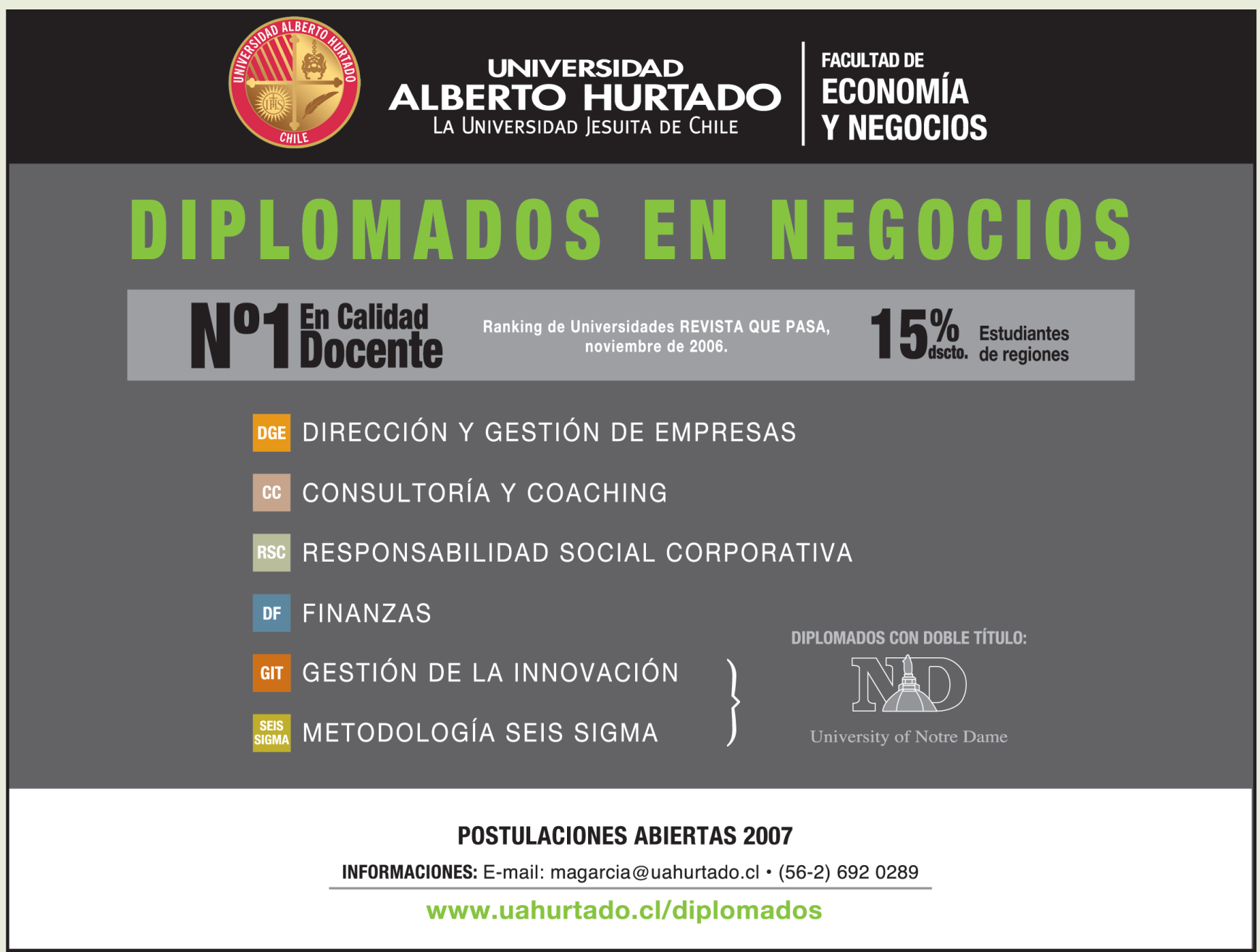

